\title{
Adjuvant transarterial chemoembolization to improve the prognosis of hepatocellular carcinoma following curative resection
}

\author{
XUEJUN KUANG ${ }^{1 *}$, JIAZHOU YE $^{2 *}$, ZHIBO XIE ${ }^{2}$, TAO BAI $^{2}$, JIE CHEN $^{2}$, WENFENG GONG $^{2}$, \\ LUNAN QI $^{2}$, JIANHONG ZHONG ${ }^{2}$, LIANG MA ${ }^{2}$, NINGFU PENG ${ }^{2}$, BANGDE XIANG $^{2}$, FEIXIANG WU ${ }^{2}$, \\ GUOBIN WU ${ }^{2}$, HAIHONG YE ${ }^{3}$, CHANGMIAO WANG ${ }^{4}$ and LUNQUN LI ${ }^{2}$ \\ ${ }^{1}$ Department of Hepatobiliary Surgery, The Affiliated Hospital of Xiangnan University, Chenzhou, Hunan 423000; \\ ${ }^{2}$ Department of Hepatobiliary and Pancreatic Surgery, Affiliated Tumor Hospital of Guangxi Medical University, \\ Nanning, Guangxi 530021; ${ }^{3}$ Department of Hepatobiliary and Pancreatic Surgery, Affiliated Minzu Hospital of \\ Guangxi Medical University, Nanning, Guangxi 530001; ${ }^{4}$ Department of General Surgery, The First \\ Hospital of Dalian Medical University, Dalian, Liaoning 116011, P.R. China
}

Received November 4, 2016; Accepted July 27, 2017

DOI: $10.3892 / \mathrm{ol} .2018 .9244$

\begin{abstract}
The present study aimed to investigate the prognostic factors for recurrence of hepatocellular carcinoma (HCC) following curative resection, and evaluate the efficacy of postoperative adjuvant transarterial chemoembolization (TACE) in improving prognosis. A total of 166 patients who underwent curative resection followed by adjuvant TACE, and 190 patients who underwent curative resection alone were studied retrospectively. Univariate and multivariate analyses were performed to investigate the risk factors of recurrence. Separated based on risk factors, subgroup studies were conducted and the association between TACE and recurrence rates was examined. Postoperative overall survival rates were determined using the Kaplan-Meier method and differences between the two therapeutic strategies were identified through log-rank analysis. Computerized tomography (CT)/magnetic resonance imaging (MRI) images were used to evaluate the function of postoperative adjuvant TACE for enhancing the efficacy of $\mathrm{CT} / \mathrm{MRI}$ in detecting recurrence. The results of the univariate and multivariate analyses revealed
\end{abstract}

Correspondence to: Dr Haihong Ye, Department of Hepatobiliary and Pancreatic Surgery, Affiliated Minzu Hospital of Guangxi Medical University, 232 Mingxiu Road, Nanning, Guangxi 530001, P.R. China

E-mail: yehaihongnn@163.com

Dr Changmiao Wang, Department of General Surgery, The First Hospital of Dalian Medical University, 222 Zhongshan Road, Dalian, Liaoning 116011, P.R. China

E-mail: wangchangmiaodl@sina.cn

*Contributed equally

Key words: hepatocellular carcinoma, curative resection, transarterial chemoembolization, recurrence that tumor capsule invasion, vascular invasion, and multiple nodules were risk factors of early recurrence. For patients with these risk factors, recurrence rates were markedly decreased at 6 and 12 months, but not at 18 and 24 months, if TACE was added to curative resection. The hepatitis $\mathrm{B}$ virus (HBV) was a risk factor for late recurrence. Postoperative TACE was not effective in reducing the recurrence rate in patients with HBV. Survival increased following curative resection with TACE compared with curative resection alone. Furthermore, adjuvant TACE enhanced the efficacy of CT/MRI in detecting recurrence. Postoperative adjuvant TACE may improve the prognosis of HCC following curative resection.

\section{Introduction}

Hepatocellular carcinoma (HCC) is one of the most common causes of cancer-associated mortality worldwide (1). For patients with resectable HCC and a moderate liver function reserve, surgical resection is an effective curative treatment (2). However, the long-term survival rates following resection remain low due to the high incidence of postoperative recurrence (3). Transarterial chemoembolization (TACE) is an adjuvant therapy commonly employed to improve the therapeutic efficacy of hepatectomy for patients with HCC (4).

A previous meta-analysis (5) demonstrated that adjuvant TACE may suppress recurrence and improve survival rates following resection. Adjuvant TACE involves the occlusion of the feeding arteries and locally administered chemotherapy, the combination of which delays tumor progression and incites tumor necrosis in HCC. In addition, the function of postoperative adjuvant TACE in improving early detection of recurrence has also been demonstrated (6). In contrast, certain studies (7) have indicated that adjuvant TACE may not significantly reduce recurrence and improve survival, and that the treatment may damage remnant liver function, particularly in patients with chronic cirrhosis (8), and that it increases the risk of hepatitis $\mathrm{B}$ virus (HBV) reactivation (9). These conflicting results leave the question concerning which subpopulations may benefit 
from adjuvant TACE following curative resection for HCC. In the present study, curative resection with and without TACE were retrospectively evaluated, to determine the efficacy of postoperative adjuvant TACE to prevent, treat and detect HCC recurrence.

\section{Patients and methods}

Patients. A total of 356 patients from the Department of Hepatobiliary and Pancreatic Surgery in the Affiliated Minzu Hospital of Guangxi Medical University (Guangxi, China) between January 2010-December 2012 were enrolled. Inclusion criteria were established at the beginning of the present study and included: i) Patients who had undergone curative resection of liver malignancies and were diagnosed with HCC by pathology; ii) the absence of apparent extrahepatic metastasis determined by preoperative and postoperative chest X-rays, computerized tomography (CT) or magnetic resonance imaging (MRI) scans; iii) complete gross resection achieved with no residual tumor in the remnant liver as determined by intraoperative visual inspection and a negatively resected margin by histological examination; iv) patients who were between 16-75 years old, who had a positive postoperative general condition as assessed by the Eastern Cooperative Oncology Group (ECOG) performance status (10) (score 0-2), and moderate liver function (Child-Pugh A) (11); v) preoperative serum levels of $\alpha$-fetoprotein (AFP) decreased to normal within 2 months following resection. The present study was approved by the Ethics Committee of Affiliated Minzu Hospital of Guangxi Medical University (Guangxi, China). Written informed consent was obtained from all participants.

Study design. A total of 166 patients who underwent curative resection followed by adjuvant TACE, and 199 patients who underwent resection alone, met the inclusion criteria and were recruited. There were four components to the present study which were as follows: i) Univariate and multivariate analyses were performed to investigate the risk factors for early (defined as recurrent HCC developing within the first year following resection) (12), or late recurrence; ii) based on recurrence risk factor analysis, subgroup studies were performed to evaluate the efficacy of suppression of recurrence by postoperative adjuvant TACE following curative resection, compared with curative resection alone; recurrence rates were compared at 6, 12, 18 and 24 months, respectively; iii) log-rank analysis was performed to compare the 1-, 2-, and 3-year overall survival between the two therapeutic strategies; iv) amongst patients who experienced recurrence, CT/MRI imaging was used to evaluate the potential function of postoperative adjuvant TACE in enhancing the efficacy of $\mathrm{CT} / \mathrm{MRI}$ in detecting recurrence.

Treatments. All patients were treated using curative resection. Surgical techniques and perioperative management were performed as previously described (13). Adjuvant TACE was performed one month following resection. With the patient under local anesthesia, contrast medium was injected into the arteries via a 4.1-5F RC1 catheter (Shanghai Medical Equipment Works Co., Ltd., Shanghai, China), which was introduced into the abdominal aorta via the right superficial femoral artery. Hepatic arterial angiography was performed using fluoroscopy to guide the catheter into the celiac and superior mesenteric arteries. Then, the feeding arteries, tumor stain, and vascular anatomy surrounding the tumor were identified. A microcatheter was introduced through the 4-5F catheter into the feeding arteries. An emulsion of 3-5 ml lipiodol and gelfoam particles with doxorubicin (30-50 mg; Shanghai Yuanye Biotechnology Co., Ltd., Shanghai, China) and cisplatin (50-100 mg; Beijing Huamaike Biotechnology Co., Ltd., Beijing, China) was infused into the feeding arteries. Postoperative adjuvant TACE was repeated for 1-6 cycles at intervals of 1 month.

Anti-virus therapy was routinely given to patients with HBV or hepatitis $\mathrm{C}$ virus following resection, as previously described (14). Percutaneous ethanol injection (PEI) or radio-ablation therapy (RA) was performed to treat recurrent HCC with a diameter of $<3 \mathrm{~cm}$. A secondary resection was only recommended when the remnant liver volume and functional reserve were considered sufficient. When patients had contradictions for, or rejected PEI, RA or secondary resection, subsequent TACE was recommended.

Monitoring for tumor recurrence and follow-up. Amongst the patients who received curative resection followed by adjuvant TACE, the serum AFP level was routinely monitored using radioimmunoassay ( ${ }^{125} \mathrm{I}$ ) (Shanghai Sangon Biological Engineering Technology And Service Co., Ltd., Shanghai, China) at the same frequency as the ultrasounds and CT/MRI scans. The process was automatically completed in GC-1200 radioimmunoassay gamma counter (Anhui Haoyuan Chemical Group Co., Ltd., Fuyang, China). Ultrasound evaluation, and dynamic CT or MRI scans were performed for surveillance of recurrence at the end of the first month following resection but prior to the first cycle of postoperative adjuvant TACE, and then at monthly intervals. If the number of adjuvant TACE cycles was $>1$, the image scan was normally performed prior to the next cycle of adjuvant TACE. Digital subtraction angiography (DSA) was performed concurrent with the adjuvant TACE. Diagnosis of recurrence was established when DSA, in addition to any one of the CT/MRI studies, was positive with or without an increase in serum AFP levels. Images from the CT/MRI scans prior to and following adjuvant TACE were compared with each other in order to evaluate whether postoperative TACE improved the efficacy of CT/MRI to discover recurrent $\mathrm{HCC}$.

Amongst patients who underwent resection alone, serum AFP level, ultrasound evaluation and dynamic CT or MRI scans were routinely monitored and performed for the surveillance of recurrence at the end of the first month subsequent to resection as well, and then at monthly intervals. DSA was performed following the diagnosis of suspected recurrence, which was detected by an increase in serum AFP level or by dynamic contrasted-enhanced CT or MRI scans. Diagnosis of recurrence was established on the basis of whether CT/MRI studies or DSA were considered as positive, with or without an increase in serum AFP level. All patients were followed up until December 30, 2013, or until mortality.

Statistical analysis. All the data were analyzed using SPSS ver. 17.0 statistical software (SPSS, Inc., Chicago, IL, USA). Normally distributed data were presented as the 
Table I. Baseline clinical and pathological characteristics of patients.

\begin{tabular}{|c|c|c|c|}
\hline Characteristics & $\begin{array}{l}\text { Curative resection }+ \text { adjuvant } \\
\text { TACE }(n=166)\end{array}$ & $\begin{array}{l}\text { Curative resection } \\
\text { alone }(\mathrm{n}=190)\end{array}$ & P-value \\
\hline \multicolumn{4}{|l|}{ Baseline characteristics } \\
\hline Age, year & $49.12 \pm 11.09$ & $51.31 \pm 11.23$ & 0.764 \\
\hline Sex, male/female & $102 / 64$ & $119 / 71$ & 0.380 \\
\hline \multicolumn{4}{|l|}{ Clinical characteristics } \\
\hline HBsAg, positive/negative & $46 / 120$ & $56 / 134$ & 0.515 \\
\hline Anti-HCV, positive/negative & $2 / 164$ & $2 / 188$ & 0.274 \\
\hline PLT, $10^{9} / 1$ & $198.25 \pm 109.17$ & $203.94 \pm 101.04$ & 0.156 \\
\hline $\mathrm{TBil}, \mu \mathrm{mol} / 1$ & $16.12(13.08-26.10)$ & $15.70(11.80-25.40)$ & 0.318 \\
\hline ALB, $g / 1$ & $37.39 \pm 3.14$ & $37.24 \pm 4.74$ & 0.936 \\
\hline ALT, U/1 & $50.00(35.00-69.00)$ & $51.01(34.00-61.00)$ & 0.837 \\
\hline AST, U/1 & $59.00(49.00-84.00)$ & $60.00(50.00-107.50)$ & 0.883 \\
\hline PT, s & $13.75 \pm 1.52$ & $13.84 \pm 1.934$ & 0.913 \\
\hline AFP, mg/l & $15.67(5.7924)$ & $12.10(1.23-26.7)$ & 0.297 \\
\hline \multicolumn{4}{|l|}{ Pathological characteristics } \\
\hline Tumor capsule, positive/negative & $40 / 126$ & $46 / 144$ & 0.101 \\
\hline Macro-vascular invasion, positive/negative & $33 / 133$ & $28 / 162$ & 0.846 \\
\hline Tumor number, single/multiple & $142 / 24$ & $136 / 54$ & 0.952 \\
\hline Tumor diameter, $\mathrm{cm}(\leq 5 / 5-10 />10)$ & $67 / 85 / 14$ & $74 / 101 / 15$ & 0.932 \\
\hline Tumor cell differentiation, high/moderate/low & $10 / 31 / 125$ & $8 / 25 / 157$ & 0.671 \\
\hline
\end{tabular}

TACE, transarterial chemoembolization; HBsAg, hepatitis B surface antigen; PLT, platelets; TBil, total bilirubin; ALB, albumin; ALT, alanine aminotransferase; AST, aspartate aminotransferase; PT, prothrombin time; AFP, $\alpha$-fetoprotein.

mean \pm standard deviation and asymmetrical distributed data were presented as the median (range). The baseline clinical and pathological characteristics for patients were analyzed using the $\chi^{2}$ test. Recurrence and survival curves were determined using the Kaplan-Meier method and differences between therapeutic strategies were identified using log-rank analysis. Prognostic factors of recurrence were evaluated by univariate and multivariate analyses using Cox's regression. $\mathrm{P}<0.05$ was considered to indicate a statistically significant difference.

\section{Results}

Baseline characteristics of patients. The baseline characteristics of all patients are summarized in Table I. No statistical difference was demonstrated in the baseline clinical and pathological variables, including age, sex, ECOG score, serum AFP level, hepatitis B surface antigen, liver function tests, tumor size, tumor capsule, tumor number, macrovascular invasion and tumor cell differentiation between patients $(\mathrm{P}>0.05)$.

Prognostic factors affecting the frequency of HCC recurrence following curative resection. During the follow-up period, 251 patients experienced early recurrence and 65 patients experienced late recurrence. Univariate analysis and multivariate analysis revealed that patients with the characteristics of tumor capsule invasion, vascular invasion, and tumor nodules had the highest tendency to develop recurrence within the first year, peaking within the first 6 months following resection. HBV was identified as a risk factor for late recurrence, primarily occurring more than 1 year following resection. Other clinicopathological characteristics including tumor size, AFP level and tumor cell differentiation did not appear to be associated with recurrence (Table II).

Effect of TACE on recurrence in risk factor subgroups. Amongst patients who had the characteristics of tumor capsule invasion, vascular invasion and multiple nodules, recurrence rates decreased significantly following curative resection when adjuvant TACE was added to therapy. This was true at 6 months (tumor capsule invasion: 23.2 vs. $59.4 \%, \mathrm{P}=0.0061$; vascular invasion: 26.3 vs. $64.5 \%, \mathrm{P}=0.0059$; multiple nodules: 12.5 vs. $31.2 \%, \mathrm{P}=0.0090$ ) and 12 months (tumor capsule invasion: 68.4 vs. $72.5 \%, \mathrm{P}=0.0093$; vascular invasion: $69.8 \%$ vs. $78.4 \%$, $\mathrm{P}=0.0067$; multiple nodules: $36.3 \%$ vs. $47.4 \%, \mathrm{P}=0.0270$ ) follow up, but not at 18 months (tumor capsule invasion: 83.1 vs. $89.2 \%, \mathrm{P}=0.2610$; vascular invasion: 87.4 vs. $91.2 \%, \mathrm{P}=0.1900$; multiple nodules: 50.3 vs. $60.1 \%, \mathrm{P}=0.0920$ ) and 24 months (tumor capsule invasion: 100.0 vs. $100.0 \%, \mathrm{P}=0.3010$; vascular invasion: 100.0 vs. $100.0 \%, \mathrm{P}=0.4080$; multiple nodules: 56.8 vs. $65.3 \%, \mathrm{P}=0.1080$; Table III and Fig. 1A-C). Amongst patients with $\mathrm{HBV}-\mathrm{HCC}$, there was no significant difference in recurrence rates at 6 months $(0.0$ vs.0.0\%, $\mathrm{P}=0.4910)$, 12 months (4.3 vs. $4.1 \%, \mathrm{P}=0.4160), 18$ months (14.6 vs. $15.3 \%, \mathrm{P}=0.6290)$, and 24 months $(22.4$ vs. $24.6 \%, \mathrm{P}=0.7500)$ following curative resection with adjuvant TACE compared with curative resection alone (Table III and Fig. 1D). These results supported the hypothesis that postoperative adjuvant TACE was effective in delaying recurrence in patients with 
Table II. Predictors of hepatocellular carcinoma recurrence.

\begin{tabular}{|c|c|c|c|c|c|c|}
\hline \multirow[b]{2}{*}{ Characteristics } & \multicolumn{4}{|c|}{ Univariate analysis } & \multicolumn{2}{|c|}{ Multivariate analysis } \\
\hline & Patients (n) & Recurrence time (month) & $95 \%$ CI & $\mathrm{P}$-value & $95 \%$ CI & P-value \\
\hline \multicolumn{7}{|l|}{ Tumor capsule } \\
\hline Positive & 270 & 5.30 & $8.37-9.16$ & $<0.001$ & $1.56-2.64$ & $<0.001$ \\
\hline Negative & 86 & 17.58 & $9.62-11.13$ & & & \\
\hline \multicolumn{7}{|c|}{ Macro-vascular invasion } \\
\hline Positive & 61 & 4.30 & $2.29-9.71$ & $<0.001$ & $0.64-3.78$ & $<0.001$ \\
\hline Negative & 295 & 19.42 & $8.60-9.14$ & & & \\
\hline \multicolumn{7}{|l|}{ Tumor number } \\
\hline Single & 278 & 17.99 & $6.74-9.85$ & $<0.001$ & $1.19-3.31$ & $<0.001$ \\
\hline Multiple & 78 & 6.96 & $9.47-10.99$ & & & \\
\hline \multicolumn{7}{|l|}{ HBsAg } \\
\hline Positive & 102 & 19.88 & $9.84-10.22$ & $<0.001$ & $1.21-3.37$ & 0.008 \\
\hline Negative & 254 & 25.28 & $7.91-9.87$ & & & \\
\hline \multicolumn{7}{|c|}{ Tumor diameter, $\mathrm{cm}$} \\
\hline$\leq 5$ & 141 & 26.48 & $7.69-9.88$ & 0.086 & $1.67-6.78$ & 0.072 \\
\hline $5-10$ & 186 & 21.82 & $10.60-11.95$ & & & \\
\hline$>10$ & 29 & 20.21 & $9.50-11.02$ & & & \\
\hline \multicolumn{7}{|c|}{ Tumor cell differentiation } \\
\hline High & 18 & 8.62 & $7.52-9.82$ & 0.091 & $1.12-3.68$ & 0.077 \\
\hline Moderate & 56 & 10.55 & $9.69-11.70$ & & & \\
\hline Low & 282 & 12.32 & $8.19-10.36$ & & & \\
\hline \multicolumn{7}{|c|}{ Postoperative TACE cycles } \\
\hline 0 & 190 & 6.58 & $5.60-9.18$ & 0.0065 & $1.06-4.86$ & 0.0029 \\
\hline 1 & 98 & 8.82 & $6.79-10.05$ & & & \\
\hline $2-3$ & 68 & 9.20 & $8.45-11.76$ & & & \\
\hline
\end{tabular}

HCC, hepatocellular carcinoma; CI, confidence interval; TACE, transarterial chemoembolization; HBsAg, hepatitis B surface antigen.

HCC with risk factors for early recurrence, but not in patients with $\mathrm{HBV}$ as a risk factor for late recurrence.

Overall survival following curative resection with and without adjuvant TACE. Overall survival increased significantly following curative resection with adjuvant TACE compared with curative resection alone. The mean survival period of patients following curative resection + TACE was 25.3 months, and the 1-, 2- and 3-year overall survival rates were 68.5, 49.3 and $27.6 \%$ respectively; the mean survival period of patients who underwent curative resection alone was 20.5 months, and the 1-, 2- and 3-year overall survival rates were 50.3, 31.2 and $20.3 \%$ respectively ( $\mathrm{P}=0.0091$; Table IV and Fig. 2). In addition, no apparent adverse events occurred in patients who underwent postoperative adjuvant TACE (Table V).

Enhancement of CT/MRI efficacy by postoperative adjuvant TACE for detecting the recurrence. During the follow-up period, amongst the patients who experienced recurrence, CT/MRI imaging was used to evaluate the potential function of postoperative adjuvant TACE in enhancing the efficacy of CT/MRI in detecting recurrence. In the present study, when the diameter of recurrent $\mathrm{HCC}$ was not $>1 \mathrm{~cm}$, nothing was discovered by dynamic contrasted-enhanced CT in the arterial enhancement period (Fig. 3A). In contrast, subsequent to adjuvant TACE being performed, a recurrent $\mathrm{HCC}$ with diameter $<1 \mathrm{~cm}$ with lipiodol uptake was revealed using non-contrast-enhanced CT (Fig. 3B). DSA was performed to make a confirmatory diagnosis of recurrence (Fig. 3C). Postoperative adjuvant TACE demonstrated a function of enhancing the efficacy of CT to detect HCCs with a diameter of $<1 \mathrm{~cm}$.

\section{Discussion}

Frequent postoperative recurrence is the main contributor to poor long-term survival following resections for HCC. The presence of non-visible intra-hepatic metastasis and multicentric occurrence prior to resection are accepted theories underlying early recurrence (15). A previous study has demonstrated that tumor capsule invasion, vascular invasion and multiple nodules occurred in association with invisible micro-metastasis (6). Unrecognized intra-hepatic metastasis had an increased likelihood of occurring in the presence of tumor capsule invasion and vascular invasion distributed via the hepatic artery and 
Table III. Hepatocellular carcinoma recurrence rates following curative resection with and without adjuvant TACE.

\begin{tabular}{|c|c|c|c|c|c|c|c|c|}
\hline \multirow{2}{*}{$\begin{array}{l}\text { Variables } \\
\text { Recurrence time, months }\end{array}$} & \multicolumn{4}{|c|}{ Curative resection + adjuvant TACE } & \multicolumn{4}{|c|}{ Curative resection alone } \\
\hline & 6 & 12 & 18 & 24 & 6 & 12 & 18 & 24 \\
\hline Tumor capsule invasion, $\%$ & 23.2 & 68.4 & 83.1 & 100.0 & 59.4 & 72.5 & 89.2 & 100.0 \\
\hline Macro-vascular invasion, $\%$ & 26.3 & 69.8 & 87.4 & 100.0 & 64.5 & 78.4 & 91.2 & 100.0 \\
\hline Multiple nodules, \% & 12.5 & 36.3 & 50.3 & 56.8 & 31.2 & 47.4 & 60.1 & 65.3 \\
\hline HBV positive, $\%$ & 0.0 & 4.3 & 14.6 & 22.4 & 0.0 & 4.1 & 15.3 & 24.6 \\
\hline
\end{tabular}

TACE, transarterial chemoembolization; HBV, hepatitis B virus.
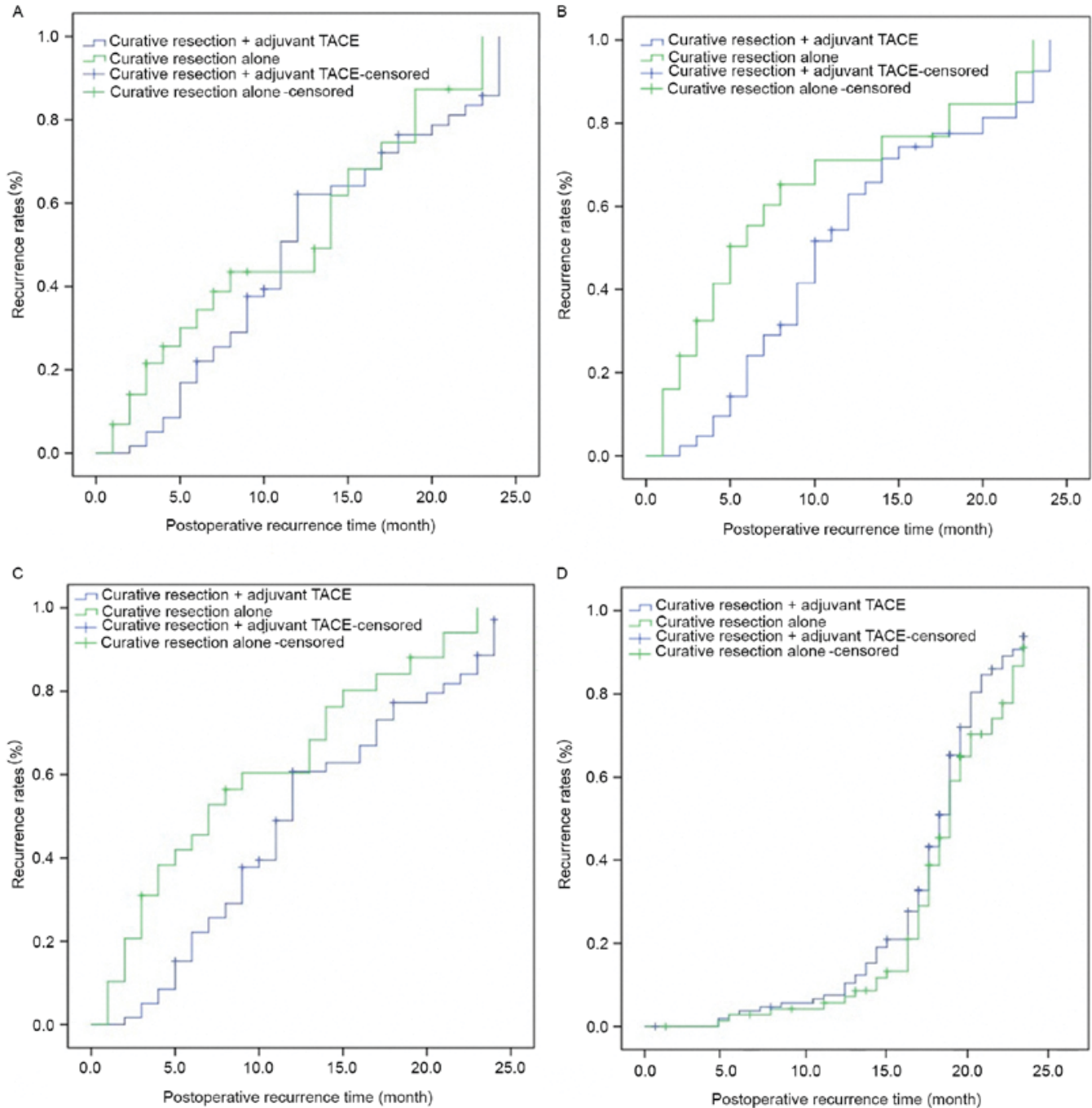

Figure 1. Recurrence curves demonstrating the difference in recurrence rate in postoperative months between groups with and without adjuvant TACE for each risk factor subgroup. Recurrence curves of subgroups of patients with hepatocellular carcinoma and (A) tumor capsule invasion, (B) macro-vascular invasion, (C) multiple nodules, and (D) hepatitis B surface antigen (positive).

portal vein system, readily escaping intra-operative detection. Additionally, from the compression and crushing of tissue during the operative process, new intra-hepatic metastases may develop. Multiple nodules were a typical outcome of the growth of previously invisible micro-metastases. Although the visible metastases were eradicated, there were still numerous invisible micro-metastases that preoperative image scans and intraoperative observation failed to detect. In this situation, liver resection was evidently not an eradicative treatment. The results of the present study had a high level of consistency with previous reports $(16,17)$. In the present retrospective study, tumor capsule invasion, vascular invasion, and multiple nodules were identified as risk factors of early recurrence. HBV was considered important for late recurrence. Preoperative serum AFP level, tumor size, and tumor cell differentiation were not associated with either early or late recurrence. 
Table IV. Mean survival periods and survival rates following curative resection with and without adjuvant TACE.

\begin{tabular}{lcccc}
\hline Treatment & $\begin{array}{c}\text { Mean survival } \\
\text { period (months) }\end{array}$ & $\begin{array}{c}\text { 1-year survival } \\
\text { rate (\%) }\end{array}$ & $\begin{array}{c}\text { 2-year survival } \\
\text { rate (\%) }\end{array}$ & $\begin{array}{c}\text { 3-year survival } \\
\text { rate }(\%)\end{array}$ \\
\hline Curative resection + adjuvant TACE & 25.3 & 68.5 & 49.3 & 27.6 \\
Curative resection alone & 20.5 & 50.3 & 31.2 & 20.3 \\
\hline
\end{tabular}

TACE, transarterial chemoembolization.

Table V. Complications and adverse events following postoperative adjuvant transarterial chemoembolization.

\begin{tabular}{lc}
\hline Complication & $\begin{array}{c}\text { No. of } \\
\text { patients (\%) }\end{array}$ \\
\hline Nausea, vomiting, n, (\%) & $29(17.5)$ \\
Fever, n (\%) & $22(13.3)$ \\
Pain, $(\%)$ & $31(18.7)$ \\
Alopecia, $\mathrm{n}(\%)$ & $3(1.8)$ \\
Liver failure, n, (\%) & $0(0.0)$ \\
Bleeding of esophageal venous plexus, n (\%) & $0(0.0)$ \\
Gastrointestinal hemorrhage & $0(0.0)$ \\
Heart failure, n (\%) & $0(0.0)$ \\
Infection, n (\%) & $0(0.0)$ \\
Ectopic embolism syndrome, n (\%) & $0(0.0)$ \\
Refractory ascites, n (\%) & $0(0.0)$ \\
Pulmonary complication, n (\%) & $1(0.6)$ \\
Therapy-related death, n (\%) & $0(0.0)$ \\
\hline
\end{tabular}

Tiny lesions have the potential to avoid preoperative or intra-operative detection. This is the primary mechanism by which micro-metastasis leads to postoperative recurrence. The normal liver parenchyma receives $\sim 70 \%$ of its basic blood supply from the portal venous system, whereas HCC growth, including the invisible micro-metastasis, depends primarily on the blood supply from the hepatic artery (18). Based on this, lipiodol may selectively accumulate in the invisible metastatic $\mathrm{HCC}$ when it is delivered intra-arterially and acts as a carrier for anticancer drugs. Additionally, TACE may effectively block the nutrient vessels of the invisible metastatic HCC, allowing sustainable chemotherapeutic killing of the microscopic HCC cells (19). Cheng et al (20) and Ren et al (21) demonstrated that postoperative adjuvant TACE protected against recurrence during the first 6 months following surgical intervention. The patients selected in these studies had incompletely encapsulated and vascular invasive tumors. The results of the present study were consistent with these reports. However, tumor necrosis in TACE is limited potentially due to ongoing nutrient transport as the tumor is fed by small nutrient vessels from the portal venous system (22). In addition, the cytotoxic effects of chemotherapy drugs that allow them to kill tumor cells follow log-cell kill kinetics (23). Presumably, therefore, the preventative efficacy would increase by repeating cycles of TACE. The present study also demonstrated that the recurrence rate

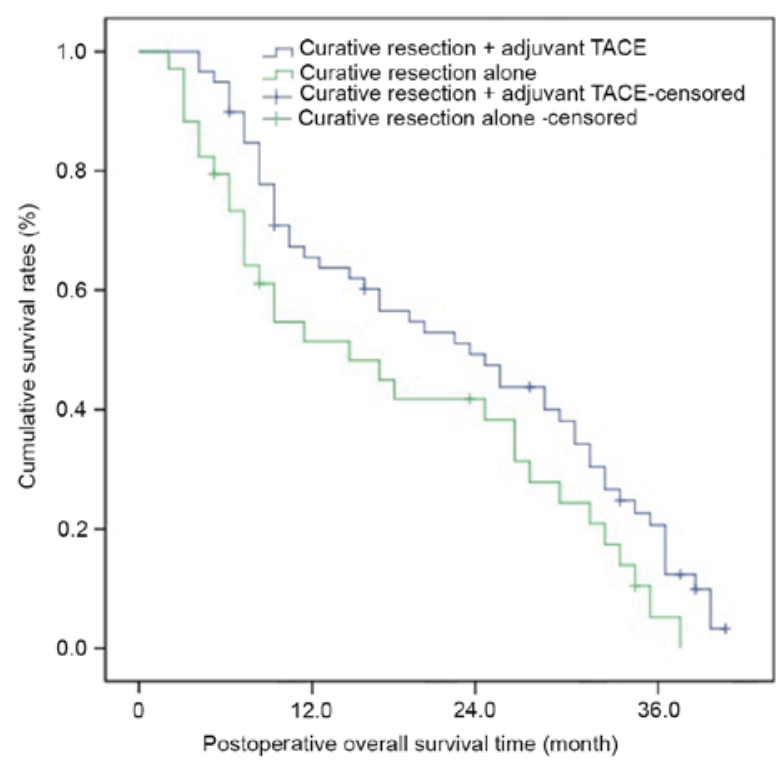

Figure 2. Postoperative cumulative survival curves of patients who underwent curative resection with/without adjuvant TACE. TACE, transarterial chemoembolization.

was significantly lower in patients who underwent 2-6 cycles of adjuvant TACE compared with patients who only received one cycle. However, repeated TACE may impair the remnant liver function following resection, particularly in patients with chronic liver cirrhosis (8), as well as an increase in the risk of HBV reactivation (9). Fortunately, the present study demonstrated that no apparent adverse events occurred in patients who underwent postoperative TACE. The results of the present study supported the recommendation of postoperative adjuvant TACE as an effective and safe treatment to delay or prevent early recurrence for patients with tumor capsule invasion, vascular invasion, or multiple nodules. The number of cycles of adjuvant TACE should depend on the remnant liver function following resection.

The efficacy of contrast-enhanced CT/MRI to detect recurrent $\mathrm{HCC}$ with diameter $<1 \mathrm{~cm}$ remains unsatisfactory. Certain studies $(6,24)$ have demonstrated that due to the exclusive uptake of lipiodol by HCC lesions, HCC-specific uptake of lipiodol would be discovered by CT or MRI scan within 3-4 weeks following lipiodol embolization. Thus, the location and size of recurrence would be identified by CT/MRI following postoperative adjuvant TACE. In the present study, in patients experiencing recurrence, contrast-enhanced CT scans were performed prior to and following adjuvant TACE and the images between the two phases were compared in order to evaluate whether this 

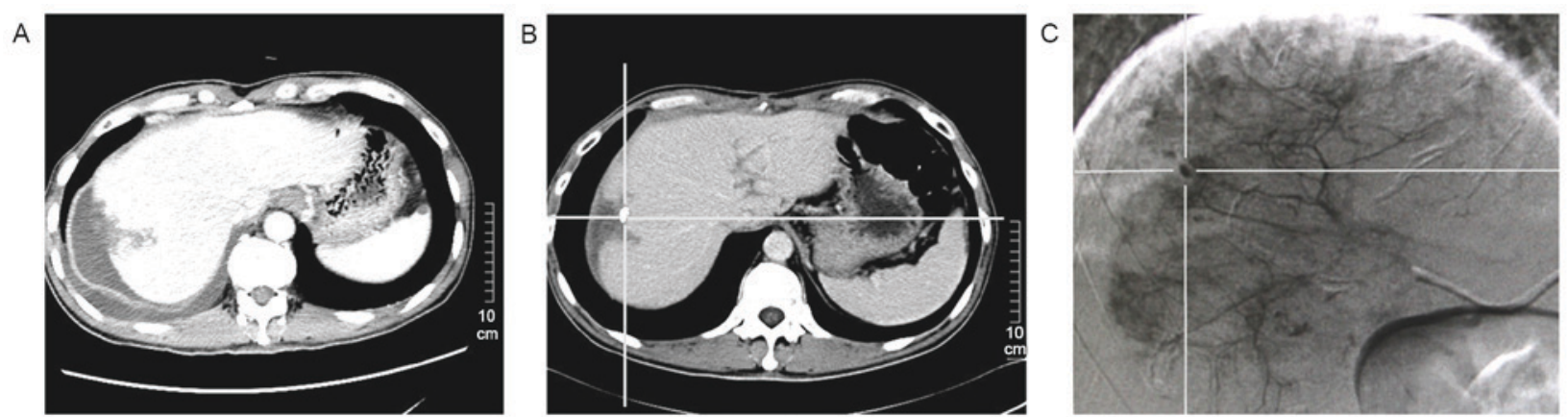

Figure 3. CT and DSA images demonstrating the effects of TACE on postoperative recurrent HCC. (A) Typical CT manifestation without TACE following curative resection. (B) Typical CT manifestation of postoperative recurrent HCC following TACE. (C) Typical DSA manifestation of postoperative HCC recurrence. $\mathrm{HCC}$, hepatocellular carcinoma; TACE, transarterial chemoembolization; CT, computerized tomography; MRI, magnetic resonance imaging; DSA, digital subtraction angiography.

strategy may improve the efficacy of CT/MRI scans to detect recurrence. DSA is favored over the CT scan for identification of small and early hepatic malignancies (6), therefore DSA was performed to confirm the CT images. The results of the present study demonstrated that when the diameter of recurrent tumor was $<1 \mathrm{~cm}$, the $\mathrm{CT}$ image following lipiodol embolization was superior to the $\mathrm{CT}$ image prior to the procedure in detecting micro recurrent HCC. The present study revealed that postoperative TACE may serve an important function in enhancing the efficacy of CT/MRI to discover the recurrence at an early stage and provide patients with optimal opportunity to receive secondary eradicative intervention like secondary resection, RA, or PEI. The clinical benefits of TACE in improving early detection of HCCs are an addition to its effect on tumor cell destruction. Postoperative preventative adjuvant TACE should be recommended in patients with higher risk of early recurrence.

When lipiodol selectively accumulates in the micro-metastasis or recurrent HCC, $>90 \%$ of tumor blood supply via the hepatic artery is blocked (15), and sustainably high levels of chemotherapeutic drugs work to kill the micro-metastatic or the recurrent HCC cells (19). Adjuvant TACE was effective in preventing early recurrence in patients with risk factors for non-visible intra-hepatic metastasis, particularly within the first 6 months following resection, and restraining the progression of recurrent HCC. Tanaka et al (25) and Izumi et al (26) reported that postoperative adjuvant TACE was effective in improving the survival of patients with HCC. The patients selected in their studies had characteristics of incomplete capsule, intra-hepatic metastasis or vascular invasion which were associated with residual tumor and earlier massive recurrence (27). The results of the present study were consistent with their reports, revealing that overall survival was significantly improved by postoperative adjuvant TACE. In addition, adjuvant TACE may enhance the efficacy of CT/MRI scans to discover micro $(<1 \mathrm{~cm})$ recurrent HCC. Early discovery of HCCs allows for an earlier opportunity to undergo eradicative interventions including secondary resection, RFA, or PEI. Generally, secondary resection, PEI and RFA as eradicative interventions are widely used to treat recurrent HCC. In contrast, adjuvant TACE has not been considered as an eradicative intervention to treat recurrent HCC because tumor necrosis is limited as stated above $(22,23)$. Although tumor necrosis may be enhanced by repeated TACE, notably, repeated TACE may damage the remnant liver parenchyma, particularly in patients with cirrhosis (8), and enhance the risk of HBV reactivation (9). Nonetheless, when recurrent HCC was $>3 \mathrm{~cm}$, or its location was adjacent to vessels, or occurred in the presence of other variables unfavorable for secondary resection, PEI or RFA, then postoperative TACE, may be a viable option as a palliative intervention to treat recurrent HCC by restraining tumor progression.

In conclusion, postoperative adjuvant TACE is an effective and safe therapeutic strategy to suppress early recurrence in patients with HCC with the characteristics of tumor capsule invasion, intra-hepatic vascular invasion and multiple nodules. Additionally, postoperative adjuvant TACE may enhance the efficacy of CT/MRI scans to discover micro-recurrence at an early stage. Furthermore, TACE may improve survival in patients with $\mathrm{HCC}$ with acceptable postoperative liver function (Child-Pugh A) by delaying tumor progression of recurrent HCCs. However, postoperative adjuvant TACE does not appear to reduce recurrence in patients without risk factors for early recurrence. Nonetheless, larger randomized control trials involving patients with more complicated cases of HCC should examine in more detail the effect of postoperative adjuvant TACE on HCC prognosis.

\section{Acknowledgements}

Not applicable.

\section{Funding}

No funding was received.

\section{Availability of data and materials}

The datasets used and/or analyzed during the present study are available from the corresponding author on reasonable request.

\section{Authors' contributions}

XK, JY, ZX, GW, FW and HY designed the study. TB, JC, WG and BX participated in data acquisition. LQ, JZ, LM, NP, $\mathrm{CW}$ and LL performed the data interpretation and statistical analyses. All authors critically revised the manuscript. 


\section{Ethics approval and consent to participate}

The present study was approved by the Ethics Committee of Affiliated Minzu Hospital of Guangxi Medical University (Guangxi, China).

\section{Patient consent for publication}

Written informed consent was obtained from all participants.

\section{Competing interests}

The authors declare that they have no competing interests.

\section{References}

1. Qin A, Zhu J, Liu X, Zeng D, Gu M and Lv C: MicroRNA-1271 inhibits cellular proliferation of hepatocellular carcinoma. Oncol Lett 14: 6783, 2017.

2. Hasegawa K, Kokudo N, Makuuchi M, Izumi N, Ichida T, Kudo M, Ku Y, Sakamoto M, Nakashima O, Matsui $\mathrm{O}$ and Matsuyama Y: Comparison of resection and ablation for hepatocellular carcinoma: A cohort study based on a Japanese nationwide survey. J Hepatol 58: 724-729, 2013.

3. Arnaoutakis DJ, Mavros MN, Shen F, Alexandrescu S, Firoozmand A, Popescu I, Weiss M, Wolfgang CL, Choti MA and Pawlik TM: Recurrence patterns and prognostic factors in patients with hepatocellular carcinoma in noncirrhotic liver: A multiinstitutional analysis. Ann Surg Oncol 21: 147-154, 2014.

4. Jiang JH, Guo Z, Lu HF, Wang XB, Yang HJ, Yang FQ, Bao SY, Zhong JH, Li LQ, Yang RR and Xiang BD: Adjuvant transarterial chemoembolization after curative resection of hepatocellular carcinoma: Propensity score analysis. World J Gastroenterol 21: 4627-4634, 2015.

5. Zhong JH and Li LQ: Postoperative adjuvant transarterial chemoembolization for participants with hepatocellular carcinoma: A meta-analysis. Hepatol Res 40: 943-953, 2010.

6. Lu X, Zhao H, Yang H, Mao Y, Sang X, Miao R, Xu Y, Du S, $\mathrm{Xu} \mathrm{H}, \mathrm{Chi} \mathrm{T}$, et al: A prospective clinical study on early recurrence of hepatocellular carcinoma after hepatectomy. J Surg Oncol 100: 488-493, 2009.

7. Chen X, Zhang B, Yin X, Ren Z, Qiu S and Zhou J: Lipiodolized transarterial chemoembolization in hepatocellular carcinoma patients after curative resection. J Cancer Res Clin Oncol 139: 773-781, 2013.

8. Ono T, Yamanoi A, Nazmy El Assal O, Kohno H and Nagasue N: Adjuvant chemotherapy after resection of hepatocellular carcinoma causes deterioration of long-term prognosis in cirrhotic patients: Metaanalysis of three randomized controlled trials. Cancer 91: 2378-2385, 2001.

9. Peng JW, Lin GN and Jiang XM: Hepatitis B virus reactivation in hepatocellular carcinoma patients underg oing transcatheterarterial chemoembolization therapy. Asia Pac J Clin Onco 8: 356-361, 2012.

10. Oken MM, Creech RH, Tormey DC, Horton J, Davis TE, McFadden ET and Carbone PP: Toxicity and response criteria of the eastern cooperative oncology group. Am J Clin Oncol 5: 649-655, 1982

11. Lin ZZ, Hsu C, Hu FC, Shao YY, Chang DY, Yang CH, Hong RL, $\mathrm{Hsu} \mathrm{CH}$ and Cheng AL: Factors impacting prognosis prediction in BCLC stage C and Child-Pugh class A hepatocellular carcinoma patients in prospective clinical trials of systemic therapy. Oncologist 17: 970-977, 2012.

12. Shah SA, Greig PD, Gallinger S, Cattral MS, Dixon E, Kim RD, Taylor BR, Grant DR and Vollmer CM: Factors associated with early recurrence after resection for hepatocellular carcinoma and outcomes. J Am Coll Surg 202: 275-283, 2006.
13. Zhong JH, Ke Y, Gong WF, Xiang BD, Ma L, Ye XP, Peng T, Xie GS and Li LQ: Hepatic resection associated with good survival for selected patients with intermediate and advanced-stage hepatocellular carcinoma. Ann Surg 260: 329-340, 2014.

14. Zhu SL, Zhong JH, Ke Y, Xiao HM, Ma L, Chen J, You XM and Li LQ: Comparative efficacy of postoperative transarterial chemoembolization with or without antiviral therapy for hepatitis B virus-related hepatocellular carcinoma. Tumour Biol 36: 6277-6284, 2015.

15. Li DH and Sun B: Research progress in intrahepatic metastasis and multiple centre carcinogenesis of hepatocellular carcinoma. Int J Surg 33: 28-31, 2006.

16. Sutcliffe R, Maguire D, Murphy P, Portmann B, Rela M, O'Sullivan G, Mufti G and Heaton N: Detection and clinical significance of bone marrow micrometastases in patients undergoing liver transplantation for hepatocellular carcinoma. Transplantation 80: 88-94, 2005.

17. Ding T, Xu J, Zhang Y, Guo RP, Wu WC, Zhang SD, Qian CN and Zheng L: Endothelium-coated tumor clusters are associated with poor prognosis and micrometastasis of hepatocellular carcinoma after resection. Cancer 117: 4878-4889, 2011.

18. Lee DS and Seong J: Radiotherapeutic options for hepatocellular carcinoma with portal vein tumor thrombosis. Liver Cancer 3: 18-30, 2014.

19. Roayaie S, Frischer JS, Emre SH, Fishbein TM, Sheiner PA, Sung M, Miller CM and Schwartz ME: Long-term results with multimodal adjuvant therapy and liver transplantation for the treatment of hepatocellular carcinomas larger than 5 centimeters. Ann Surg 235: 533-539, 2002.

20. Cheng HY, Wang X, Chen D, Xu AM and Jia YC: The value and limitation of transcatheter arterial chemoembolization in preventing recurrence of resected hepatocellular carcinoma. World J Gastroenterol 11: 3644-3646, 2005.

21. Ren ZG, Lin ZY, Xia JL, Ye SL, Ma ZC and Ye QH: Postoperative adjuvant arterial chemoembolization improves survival of hepatocellular carcinoma patients with risk factors for residual tumor: A retrospective control study. World J Gastroenterol 10: 2791-2794, 2004.

22. Luo J, Guo RP, Lai EC, Zhang YJ, Lau WY, Chen MS and Shi M: Transarterial chemoembolization for unresectable hepatocellular carcinoma with portal vein tumor thrombosis: A prospective comparative study. Ann Surg Oncol 18: 413-420, 2011.

23. Norton L: Adjuvant breast cancer therapy: Current status and future strategies-growth kinetics and the improved drug therapy of breast cancer. Semin Oncol 26 (1 Suppl 3): S1-S4, 1999.

24. Dai D, Xu W, Liu J, Zhu L, Zhu X and Ma X: Safety and efficacy of a peripheral intravenous bolus of Licartin for the treatment of advanced hepatocellular carcinoma. Exp Ther Med 6: 1417-1422, 2013.

25. Tanaka K, Shimada H, Togo S, Takahashi T, Endo I and Sekido H: Use of transcatheter arterial infusion of anticancer agents with lipiodol to prevent recurrence of hepatocellular carcinoma after hepatic resection. Hepatogastroenterology 46: 1083-1088, 1999.

26. Izumi R, Shimizu K and Miyazaki I: Postoperative adjuvant locoregional chemotherapy in patients with hepatocellular carcinoma. Hepatogastroenterology 43: 1415-1420, 1996.

27. Yamanaka J, Yamanaka N, Nakasho K, Tanaka T, Ando T, Yasui C, Kuroda N, Takata M, Maeda S, Matsushita K, et al: Clinicopathologic analysis of stage II-III hepatocellular carcinoma showing early massive recurrence after liver resection. J Gastroenterol Hepatol 15: 1192-1198, 2000.

This work is licensed under a Creative Commons Attribution-NonCommercial-NoDerivatives 4.0 International (CC BY-NC-ND 4.0) License. 